\title{
Towards a Worldsheet Derivation of the Maldacena Conjecture
}

\section{Citation}

Berkovits, Nathan, and Cumrun Vafa. 2008. Towards a Worldsheet Derivation of the Maldacena Conjecture. Journal of High Energy Physics 2008(03): 031.

\section{Published Version}

doi:10.1088/1126-6708/2008/03/031

\section{Permanent link}

http://nrs.harvard.edu/urn-3:HUL.InstRepos:8152135

\section{Terms of Use}

This article was downloaded from Harvard University's DASH repository, and is made available under the terms and conditions applicable to Open Access Policy Articles, as set forth at http:// nrs.harvard.edu/urn-3:HUL.InstRepos:dash.current.terms-of-use\#OAP

\section{Share Your Story}

The Harvard community has made this article openly available.

Please share how this access benefits you. Submit a story.

\section{Accessibility}


IFT-P.018/2007

\title{
Towards a Worldsheet Derivation of the Maldacena Conjecture
}

\author{
Nathan Berkovits] \\ Instituto de Física Teórica, State University of São Paulo \\ Rua Pamplona 145, 01405-900, São Paulo, SP, Brasil \\ and \\ Cumrun Vafal \\ Jefferson Laboratory, Harvard University \\ Cambridge, MA 02138, USA
}

\begin{abstract}
A $U(2,2 \mid 4)$-invariant A-model constructed from fermionic superfields has recently been proposed as a sigma model for the superstring on $A d S_{5} \times S^{5}$. After explaining the relation of this A-model with the pure spinor formalism, the A-model action is expressed as a gauged linear sigma model. In the zero radius limit, the Coulomb branch of this sigma model is interpreted as D-brane holes which are related to gauge-invariant $\mathcal{N}=4 \mathrm{~d}=4$ super-YangMills operators. As in the worldsheet derivation of open-closed duality for Chern-Simons theory, this construction may lead to a worldsheet derivation of the Maldacena conjecture. Intriguing connections to the twistorial formulation of $\mathcal{N}=4$ Yang-Mills are also noted.
\end{abstract}

November 2007

\footnotetext{
1 e-mail: nberkovi@ift.unesp.br

2 e-mail: vafa@physics.harvard.edu
} 


\section{Introduction}

Large $N$ dualities between gauge theories and gravity have been an important development in our understanding of string theory. In particular a large collection of D-branes can be equivalently described by a dual purely gravitational system which the D-branes generate. A prominent example of this [1] is the duality between the gauge system living on $N$ D3 branes in the $\alpha^{\prime} \rightarrow 0$ limit (i.e., $\mathcal{N}=4$ supersymmetric $U(N)$ Yang-Mills in $d=4)$ and the dual $A d S_{5} \times S^{5}$ where the D-branes have been replaced with flux.

From the worldsheet perspective the duality can be interpreted as follows: Let $\lambda$ denote the string coupling constant. For each genus $g$ in perturbation theory, on the Dbrane side we have to insert an arbitrary number of holes $h$ ending on the D-branes. This gives rise to the factor $N^{h}$ for such amplitudes. In addition this diagram is weighted with $\lambda^{2 g-2+h}$. Thus altogether we have a factor

$$
F_{g, h} \lambda^{2 g-2+h} N^{h} .
$$

We consider first summing over the number of holes. Replacing $N \lambda=T$, the 't Hooft parameter, we have

$$
\lambda^{2 g-2} \sum_{h} F_{g, h} T^{h}=\lambda^{2 g-2} F_{g}
$$

where

$$
F_{g}(T)=\sum_{h} F_{g, h} T^{h}
$$

is interpreted as the genus $g$ correction of a dual gravitational system where $T$ plays the role of a modulus in the gravitational dual. In other words the large $N$ duality is a statement that can be seen order by order in closed string pertubation theory. The subtlety is only that the effective open string coupling $N \lambda=T$ can be large. For large $T$ the gravitational description is the better description and for small $T$ the gauge theory description, involving D-branes.

One idea for a perturbative proof of the Maldacena conjecture would thus involve showing that if we start with the closed string description of the system and take $T \rightarrow 0$ the worldsheet description will develop two phases $(\mathrm{H}, \mathrm{C})$, in one of which $(\mathrm{C})$ the degrees of freedom are frozen out. Viewed from the perspective of the $H$ system we thus have holes where the worldsheet is in the $(\mathrm{C})$ phase. One has to show that the amplitudes are non-vanishing only if the $(\mathrm{C})$ phase has the topology of a disc and that the path-integral on each $(\mathrm{C})$ region gives the correct factor of $N \lambda$. This idea, which was suggested in [2] 
in the context of large $N$ duality between $U(N)$ Chern-Simons theory on $S^{3}$ and resolved conifold geometry, was implemented in [3] and also applied to derivations of duality for the F-terms in its superstring embedding [4]. Prominent in this derivation was the rewriting of a topological A-model in the form of a linear sigma model and identifying the two phases as $H=$ Higgs and $C=$ Coulomb branches of the sigma model as the modulus $T$ of the closed string approaches zero. The aim of the present paper is to propose a similar scenario for the large $N$ duality of $\mathcal{N}=4$ Yang-Mills and $A d S_{5} \times S^{5}$.

A basic step in this direction has already been taken [5]. In particular it was shown that the gravity side, i.e. type IIB superstrings on $A d S_{5} \times S^{5}$ geometry, can be viewed as an A-model topological string on the coset $\frac{U(2,2 \mid 4)}{U(2,2) \times U(4)}$. Here we make this map more precise and furthermore recast it as a gauged linear sigma model. In this formulation, as the closed string modulus approaches zero, once again we obtain two branches. We will argue, just as in the Chern-Simons case, that the Coulomb branch corresponds to holes in this formulation. We thus end up with a worldsheet with an arbitrary number of holes, which can then be interpreted as the 't Hooft diagrams of $\mathcal{N}=4$ supersymmetric $U(N)$ Yang-Mills theory. As evidence for this derivation we show how the half BPS sector of the two sides map to one another in this setup. In addition we find an intriguing connection with the twistorial formulation of $\mathcal{N}=4$ Yang-Mills: a generic point on the Coulomb branch of the linear sigma model gives four copies of $\mathbf{C P}^{3 \mid 4}$. Even though we do not exploit this connection it is rather suggestive.

The organization of this paper is as follows: In section 2 we review the A-model formulation of the $A d S_{5} \times S^{5}$. In section 3, the relation between this A-model formulation and the pure spinor fomulation of $A d S_{5} \times S^{5}$ is clarified. In section 4 we review the derivation of the large $N$ duality between Chern-Simons and topological strings on the resolved conifold. In section 5 we construct the gauged linear sigma model and propose a large $N$ derivation for our sigma model. In section 6 we discuss our conclusions and open questions.

\section{Review of A-Model}

\subsection{Worldsheet variables}

In [5], an $\mathrm{N}=2$ worldsheet supersymmetric A-model was conjectured to describe the superstring on $A d S_{5} \times S^{5}$. Instead of being constructed using the $\frac{P S U(2,2 \mid 4)}{S O(4,1) \times S O(5)}$ supercoset 
of Metsaev-Tseytlin, the variables in the A-model are described by $\mathrm{N}=2$ worldsheet super-

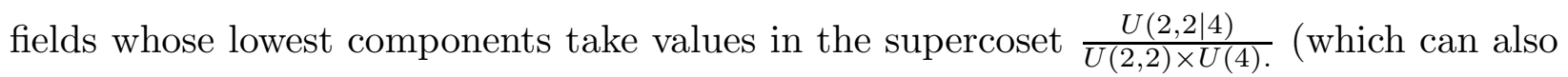
be expressed as $\frac{P U(2,2 \mid 4)}{S U(2,2) \times U(4)}$ or $\left.\frac{P S U(2,2 \mid 4)}{S U(2,2) \times S U(4)}\right)$. Since this supercoset only has fermionic elements, the worldsheet superfields are all fermionic and will be called $\Theta_{J}^{A}$ and $\bar{\Theta}_{A}^{J}$ where $A=1$ to 4 and $J=1$ to 4 label fundamental representations of $U(2,2)$ and $U(4)$ respectively. Furthermore, $\Theta_{J}^{A}$ and $\bar{\Theta}_{A}^{J}$ will be defined to be $\mathrm{N}=2$ chiral and antichiral superfields with the component expansions

$$
\begin{aligned}
& \Theta_{J}^{A}\left(\kappa_{+}, \kappa_{-}\right)=\theta_{J}^{A}+\kappa_{+} Z_{J}^{A}+\kappa_{-} \bar{Y}_{J}^{A}+\kappa_{+} \kappa_{-} f_{J}^{A}, \\
& \bar{\Theta}_{A}^{J}\left(\bar{\kappa}_{+}, \bar{\kappa}_{-}\right)=\bar{\theta}_{A}^{J}+\bar{\kappa}_{+} \bar{Z}_{A}^{J}+\bar{\kappa}_{-} Y_{A}^{J}+\bar{\kappa}_{+} \bar{\kappa}_{-} \bar{f}_{A}^{J},
\end{aligned}
$$

where $\left(\kappa_{+}, \bar{\kappa}_{+}\right)$are left-moving and $\left(\kappa_{-}, \bar{\kappa}_{-}\right)$are right-moving Grassmann parameters, and $f_{J}^{A}$ and $\bar{f}_{A}^{J}$ are auxiliary fields.

As discussed in [5], the 32 variables $\theta_{J}^{A}$ and $\bar{\theta}_{A}^{J}$ are related to the usual 32 fermionic variables of $A d S_{5} \times S^{5}$ superspace, whereas the 32 bosonic variables $Z_{J}^{A}$ and $\bar{Z}_{A}^{J}$ are twistorlike combinations of the 10 spacetime variables $x^{M}$ and the 22 pure spinor ghost variables $\left(\lambda^{\alpha}, \bar{\lambda}^{\widehat{\alpha}}\right)$ of the pure spinor formalism. Note that $d=10$ spacetime vectors will be denoted using either the index $M=0$ to 9 or the $A d S_{5} \times S^{5}$ indices $(m, \tilde{m})=1$ to 5 . And $d=10$ spacetime spinors will be denoted using either the index $\alpha=1$ to 16 or $\widehat{\alpha}=1$ to 16 depending if, in a flat background, the spacetime spinors are left or right-moving on the worldsheet.

To express $Z_{J}^{A}$ and $\bar{Z}_{A}^{J}$ in terms of $(x, \lambda, \bar{\lambda})$, first parameterize the $A d S_{5}$ variable $x^{m}$ for $m=1$ to 5 as an $\frac{S U(2,2)}{S O(4,1)}$ coset $H_{A^{\prime}}^{A}(x)$ where $A^{\prime}=1$ to 4 is an $S O(4,1)$ spinor index, and parameterize the $S^{5}$ variable $\tilde{x}^{\tilde{m}}$ for $\tilde{m}=1$ to 5 as an $\frac{S U(4)}{S O(5)} \operatorname{coset} \tilde{H}_{J^{\prime}}^{J}(\tilde{x})$ where $J^{\prime}=1$ to 4 is an $S O(5)$ spinor index. Writing the $S O(9,1)$ spinor index in terms of these $S O(4,1) \times S O(5)$ spinor indices, the left and right-moving pure spinor variables $\lambda^{\alpha}$ and $\bar{\lambda}^{\widehat{\alpha}}$ satisfying $\lambda \gamma^{M} \lambda=0$ and $\bar{\lambda} \gamma^{M} \bar{\lambda}=0$ decompose as $\lambda_{J^{\prime}}^{A^{\prime}}$ and $\bar{\lambda}_{A^{\prime}}^{J^{\prime}}$ which satisfy

$$
\begin{aligned}
& \lambda_{J^{\prime}}^{A^{\prime}} \sigma_{A^{\prime} B^{\prime}}^{m}\left(\tilde{\sigma}^{6}\right)^{J^{\prime} K^{\prime}} \lambda_{K^{\prime}}^{B^{\prime}}=\lambda_{J^{\prime}}^{A^{\prime}} \sigma_{A^{\prime} B^{\prime}}^{6}\left(\tilde{\sigma}^{\tilde{m}}\right)^{J^{\prime} K^{\prime}} \lambda_{K^{\prime}}^{B^{\prime}}=0, \\
& \bar{\lambda}_{A^{\prime}}^{J^{\prime}}\left(\sigma^{m}\right)^{A^{\prime} B^{\prime}} \tilde{\sigma}_{J^{\prime} K^{\prime}}^{6} \bar{\lambda}_{B^{\prime}}^{K^{\prime}}=\bar{\lambda}_{A^{\prime}}^{J^{\prime}}\left(\sigma^{6}\right)^{A^{\prime} B^{\prime}} \tilde{\sigma}_{J^{\prime} K^{\prime}}^{\tilde{m}} \bar{\lambda}_{B^{\prime}}^{K^{\prime}}=0,
\end{aligned}
$$

where $\left(\sigma_{A^{\prime} B^{\prime}}^{m}, \sigma_{A^{\prime} B^{\prime}}^{6}\right)$ are the six Pauli matrices for $S O(4,2)=S U(2,2)$ and $\left(\tilde{\sigma}_{J^{\prime} K^{\prime}}^{\tilde{m}}, \tilde{\sigma}_{J^{\prime} K^{\prime}}^{6}\right)$ are the six Pauli matrices for $S O(6)=S U(4)$. Note that $S O(4,1) \times S O(5)$ spinor indices 
can be raised and lowered using $\sigma_{A^{\prime} B^{\prime}}^{6}$ and $\tilde{\sigma}_{J^{\prime} K^{\prime}}^{6}$, however, it will be convenient to always write $\lambda^{\alpha}$ and $\bar{\lambda}^{\widehat{\alpha}}$ as $\lambda_{J^{\prime}}^{A^{\prime}}$ and $\bar{\lambda}_{A^{\prime}}^{J^{\prime}}$.

The twistor-like combinations $Z_{J}^{A}$ and $\bar{Z}_{A}^{J}$ are constructed from these $x$ 's and $\lambda$ 's as

$$
Z_{J}^{A}=H_{A^{\prime}}^{A}(x)\left(\tilde{H}^{-1}(\tilde{x})\right)_{J}^{J^{\prime}} \lambda_{J^{\prime}}^{A^{\prime}}, \quad \bar{Z}_{A}^{J}=\left(H^{-1}(x)\right)_{A}^{A^{\prime}} \tilde{H}_{J^{\prime}}^{J}(\tilde{x}) \bar{\lambda}_{A^{\prime}}^{J^{\prime}}
$$

Since $\left(x^{m}, \tilde{x}^{\tilde{m}}\right)$ and $\left(\lambda^{\alpha}, \bar{\lambda}^{\widehat{\alpha}}\right)$ contain 32 independent components and since $Z_{J}^{A}$ and $\bar{Z}_{A}^{J}$ are unconstrained, the construction of (2.3) is invertible for generic values of $(x, \tilde{x})$ and $(\lambda, \bar{\lambda})$. So for generic values of $\left(Z_{J}^{A}, \bar{Z}_{A}^{J}\right)$, the inverse map of (2.3) gives a point $\left(x^{m}, \tilde{x}^{\tilde{m}}\right)$ on $A d S_{5} \times S^{5}$ together with a pair of pure spinors $\left(\lambda^{\alpha}, \bar{\lambda}^{\widehat{\alpha}}\right)$. In $\mathrm{d}=10$ Euclidean space, one can treat $\bar{\lambda}_{\alpha} \equiv\left(\gamma^{01234}\right)_{\alpha \widehat{\alpha}} \bar{\lambda}^{\widehat{\alpha}}$ as the complex conjugate of $\lambda^{\alpha}$, which implies that $\bar{Z}_{A}^{J}$ is the complex conjugate of $Z_{J}^{A}$.

\subsection{Worldsheet action}

As discussed in [5], the $U(2,2 \mid 4)$-invariant action for the A-model can be written in $\mathrm{N}=(2,2)$ superspace as

$$
S=t \int d^{2} z \int d^{4} \kappa \operatorname{Tr}\left[\log \left(\delta_{K}^{J}+\bar{\Theta}_{A}^{J} \Theta_{K}^{A}\right)\right]
$$

where $t$ is a constant parameter and the notation $\log \left(M_{K}^{J}\right)$ denotes the matrix $(\log M)_{K}^{J}$. The bosonic $U(2,2) \times U(4)$ isometries act in the obvious way as

$$
\delta \Theta_{J}^{A}=i \Lambda_{B}^{A} \Theta_{J}^{B}+i \tilde{\Lambda}_{J}^{K} \Theta_{K}^{A}, \quad \delta \bar{\Theta}_{A}^{J}=-i \Lambda_{A}^{B} \bar{\Theta}_{B}^{J}-i \tilde{\Lambda}_{K}^{J} \bar{\Theta}_{A}^{K}
$$

and the 32 fermionic isometries act nonlinearly as

$$
\delta \Theta_{J}^{A}=\epsilon_{J}^{A}+\Theta_{K}^{A} \bar{\epsilon}_{B}^{K} \Theta_{J}^{B}, \quad \delta \bar{\Theta}_{A}^{J}=\bar{\epsilon}_{A}^{J}+\bar{\Theta}_{B}^{J} \epsilon_{K}^{B} \bar{\Theta}_{A}^{K}
$$

One can easily check that under the fermionic isometries, $\delta \operatorname{Tr}\left[\log \left(\delta_{K}^{J}+\bar{\Theta}_{A}^{J} \Theta_{K}^{A}\right)\right]=\bar{\epsilon}_{A}^{J} \Theta_{J}^{A}+$ $\bar{\Theta}_{A}^{J} \epsilon_{J}^{A}$, and since $\Theta_{J}^{A}$ and $\bar{\Theta}_{A}^{J}$ are chiral and antichiral, the action of (2.4) is invariant.

After integrating out the auxiliary fields $f_{J}^{A}$ and $\bar{f}_{A}^{J}$, the action of (2.4) can be written in terms of the component fields of (2.1) as

$$
\begin{gathered}
S=t \int d^{2} z\left[\left(G^{-1} \partial G\right)_{J}^{A}\left(G^{-1} \bar{\partial} G\right)_{A}^{J}\right. \\
\left.-Y_{A}^{J}(\bar{\nabla} Z)_{J}^{A}+\bar{Y}_{J}^{A}(\nabla \bar{Z})_{A}^{J}+(Y Z)_{K}^{J}(\overline{Z Y})_{J}^{K}-(Z Y)_{B}^{A}(\overline{Y Z})_{A}^{B}\right]
\end{gathered}
$$


where $G(\theta, \bar{\theta})$ takes values in the fermionic coset $\frac{U(2,2 \mid 4)}{U(2,2) \times U(4)}$ which has 32 fermionic parameters, $\left(G^{-1} \partial G\right)$ and $\left(G^{-1} \bar{\partial} G\right)$ are the left-invariant currents taking values in the Lie algebra of $U(2,2 \mid 4),(Y Z)_{K}^{J}=Y_{A}^{J} Z_{K}^{A},(Z Y)_{B}^{A}=Z_{K}^{A} Y_{B}^{K}$, and

$$
\begin{aligned}
& (\bar{\nabla} Z)_{J}^{A}=\bar{\partial} Z_{J}^{A}+\left(G^{-1} \bar{\partial} G\right)_{B}^{A} Z_{J}^{B}-\left(G^{-1} \bar{\partial} G\right)_{J}^{K} Z_{K}^{A} \\
& (\nabla \bar{Z})_{A}^{J}=\partial \bar{Z}_{A}^{J}-\left(G^{-1} \partial G\right)_{A}^{B} \bar{Z}_{B}^{J}+\left(G^{-1} \bar{\partial} G\right)_{K}^{J} \bar{Z}_{A}^{K}
\end{aligned}
$$

Note that $\mathrm{N}=(2,2)$ worldsheet supersymmetry is manifest using the superspace form of the action of (2.4), whereas $U(2,2 \mid 4)$ symmetry is manifest using the component form of the action of (2.7). As will be shown in section 5, both these symmetries can be made manifest by writing the action as a gauged linear sigma model. Furthermore, it was shown in [5] that this A-model action has no conformal anomaly.

\subsection{Open string sector}

As discussed in [5], a natural open string boundary condition for the A-model is

$$
\bar{\Theta}_{A}^{J}=\delta^{J K} \epsilon_{A B} \Theta_{K}^{B}
$$

where $\epsilon_{A B}$ is an antisymmetric tensor which breaks $S U(2,2)$ to $S O(3,2)$. The boundary condition of (2.9) is similar to the open string boundary condition for the Chern-Simons topological string which is $\bar{X}_{I}=\delta_{I J} X^{J}$ for $I, J=1$ to 3 . Note that the open string boundary for the A-model is defined by $z=\bar{z}, \kappa_{+}=\bar{\kappa}_{-}$, and $\bar{\kappa}_{+}=\kappa_{-}$, so (2.9) implies that

$$
\bar{\theta}_{A}^{J}=\delta^{J K} \epsilon_{A B} \theta_{K}^{B}, \quad \bar{Z}_{A}^{J}=\delta^{J K} \epsilon_{A B} Z_{K}^{B}, \quad Y_{A}^{J}=\delta^{J K} \epsilon_{A B} \bar{Y}_{K}^{B}
$$

The boundary condition of (2.9) breaks half of the fermionic isometries and reduces the $U(2,2 \mid 4)$ supergroup of isometries to the supergroup $O S p(4 \mid 4)$. This supergroup contains $S O(3,2) \times S O(4)$ bosonic isometries and 16 fermionic isometries, and is the $\mathcal{N}=4$ supersymmetry algebra on $A d S_{4}$.

In [5], it was conjectured that the open string sector of the A-model might describe $\mathcal{N}=4 \mathrm{~d}=4$ super-Yang-Mills in the same manner that the open sector of Witten's topological A-model describes $d=3$ Chern-Simons. Evidence for this conjecture came from the fact that the $\alpha^{\prime} \rightarrow 0$ limit of this open string sector is described by the pure spinor superparticle whose spectrum is $\mathcal{N}=4 \mathrm{~d}=4$ super-Yang-Mills. However, it was not proven 
that there are no massive states in the open string sector coming from the worldsheet nonzero modes.

In this paper, the conjecture that the open string sector of the A-model contains only $\mathcal{N}=4 \mathrm{~d}=4$ super-Yang-Mills states will be withdrawn, and it will instead be argued that the open string boundary conditions of (2.9) describe an $A d S_{4}$ D-brane probe embedded in $A d S_{5} \times S^{5}$. Although the low-energy sector of this D-brane probe contains $\mathcal{N}=4 \mathrm{~d}=4$ super-Yang-Mills states, one also expects to have massive states in the spectrum. Note that the position of this $A d S_{4}$ D-brane probe in $A d S_{5}$ is determined by the choice of the

antisymmetric tensor $\epsilon_{A B}$ in (2.9). There are $\frac{S O(4,2)}{S O(3,2)}$ different ways to embed $A d S_{4}$ in $A d S_{5}$, and the choice of $\epsilon_{A B}$ determines this embedding.

\section{Relation of A-model with Pure Spinor Formalism}

In this section, the relation between the A-model action of (2.4) and the pure spinor $A d S_{5} \times S^{5}$ sigma model will be clarified. (In [5], the relation between these actions was understood only in a certain singular limit of the superspace torsion.) Using the field redefinition of (2.3), it will be shown that the A-model maps into the pure spinor sigma model where the parameter $t$ in (2.4) is related to the $A d S_{5}$ radius $R$ as $t=\frac{1}{2} R^{2}$. When $t \rightarrow \infty$, the A-model becomes weakly coupled and describes the flat-space limit of the $A d S_{5} \times S^{5}$ sigma model. And when $t \rightarrow 0$, the A-model becomes strongly coupled and describes the highly curved limit of the $A d S_{5} \times S^{5}$ sigma model. As will be discussed in section 5 , much can be learned about the $t \rightarrow 0$ limit by writing the A-model as a gauged linear sigma model.

Although the A-model of (2.4) is invariant under $U(2,2 \mid 4)$ global isometry, the pure spinor $A d S_{5} \times S^{5}$ sigma model (like the Green-Schwarz $A d S_{5} \times S^{5}$ sigma model) is only invariant under $P S U(2,2 \mid 4)$ isometry. Nevertheless, it will be shown in subsection (3.1) that after adding a BRST-trivial term, the pure spinor sigma model can be expressed as a $U(2,2 \mid 4)$-invariant action. The field redefinition of (2.3) will then be used in subsection (3.2) to map this $U(2,2 \mid 4)$ invariant form of the pure spinor sigma model into the A-model action of (2.4).

Since the physical theory described by the sigma model is invariant under only $P S U(2,2 \mid 4)$ isometry, a natural question is how the bonus $\mathrm{U}(1)$ symmetry is broken. (Note that one of the $U(1)$ 's in $U(2,2 \mid 4)$ acts trivially on all fields. The "bonus" $U(1)$ is the symmetry in $P U(2,2 \mid 4)$ which is not in $P S U(2,2 \mid 4)$.) As will be discussed in subsection (3.3), the bonus $U(1)$ symmetry is preserved by the worldsheet action but will be broken by the BRST operator which determines the physical state conditions. 


\section{1. $U(2,2 \mid 4)$-invariant pure spinor sigma model}

Using the conventions of [6], the pure spinor sigma model action is

$$
\begin{gathered}
S=R^{2} \int d^{2} z\left[\frac{1}{2} \eta_{M N} J^{M} \bar{J}^{N}-\eta_{\alpha \widehat{\beta}}\left(\frac{3}{4} J^{\widehat{\beta}} \bar{J}^{\alpha}+\frac{1}{4} \bar{J}^{\widehat{\beta}} J^{\alpha}\right)\right. \\
\left.-w_{\alpha} \bar{\nabla} \lambda^{\alpha}+\bar{w}_{\widehat{\alpha}} \nabla \bar{\lambda}^{\widehat{\alpha}}-\frac{1}{4} \eta_{[M N][P Q]}\left(w \gamma^{[M N]} \lambda\right)\left(\bar{w} \gamma^{[P Q]} \bar{\lambda}\right)\right]
\end{gathered}
$$

where

$$
\bar{\nabla} \lambda^{\alpha}=\left(\bar{\partial} \lambda+\frac{1}{2} J^{[M N]} \gamma_{M N} \lambda\right)^{\alpha}, \quad \nabla \bar{\lambda}^{\widehat{\alpha}}=\left(\partial \bar{\lambda}+\bar{J}^{[M N]} \gamma_{[M N]} \bar{\lambda}\right)^{\widehat{\alpha}}
$$

and $J=\left(g^{-1} \partial g\right)$ and $\bar{J}=\left(g^{-1} \bar{\partial} g\right)$ are the Metsaev-Tseytlin left-invariant currents constructed from a matrix $g(x, \theta, \bar{\theta})$ taking values in the supercoset $\frac{P S U(2,2 \mid 4)}{S O(4,1) \times S O(5)}$. These currents $J$ take values in the $P S U(2,2 \mid 4)$ Lie algebra where $J^{M}=\left(J^{m}, J^{\tilde{m}}\right)$ are the 10 translation currents, $J^{\alpha}$ and $J^{\widehat{\alpha}}$ are the 32 supersymmetry currents, and $J^{[M N]}=\left(J^{[m n]}, J^{[\tilde{m} \tilde{n}]}\right)$ are the $20 S O(4,1) \times S O(5)$ Lorentz currents. Furthermore, $\eta_{\alpha \widehat{\beta}}=\left(\gamma^{01234}\right)_{\alpha \widehat{\beta}}, \eta_{[m n][p q]}=$ $\eta_{m[p} \eta_{q] n}$ and $\eta_{[\tilde{m} \tilde{n}][\tilde{p} \tilde{q}]}=-\eta_{\tilde{m}[\tilde{p}} \eta_{\tilde{q}] \tilde{n}}$.

Under the "bonus" $U(1)$ symmetry of $P U(2,2 \mid 4), J^{\alpha}$ and $J^{\widehat{\alpha}}$ rotate into each other as

$$
\delta J^{\alpha}=i J^{\widehat{\alpha}}, \quad \delta J^{\widehat{\alpha}}=-i J^{\alpha}
$$

In other words, $\left(J^{\alpha} \pm i J^{\widehat{\alpha}}\right)$ carries $\pm U(1)$ charge under this symmetry. Since $J^{M}$ and $J^{[M N]}$ are $U(1)$ invariant, the action of (3.1) transforms under the bonus $U(1)$ as

$$
\delta S=R^{2} \int d^{2} z\left[-i \eta_{\alpha \beta} J^{\beta} \bar{J}^{\alpha}+i \eta_{\widehat{\alpha} \widehat{\beta}} J^{\widehat{\beta}} \bar{J}^{\widehat{\alpha}}\right]
$$

where $\eta_{\alpha \beta}=\left(\gamma^{01234}\right)_{\alpha \beta}$ and $\eta_{\widehat{\alpha} \widehat{\beta}}=\left(\gamma^{01234}\right)_{\widehat{\alpha} \widehat{\beta}}$. Nevertheless, by adding a BRST-trivial term to the action, this $U(1)$ transformation can be cancelled. The resulting $U(2,2 \mid 4)$ invariant action can then be mapped into the A-model action of (2.4).

The BRST-trivial term is given by

$$
\begin{gathered}
S_{\text {trivial }}=-\frac{1}{2} R^{2} \int d^{2} z\left[\frac{1}{2} \frac{\eta^{\alpha \widehat{\beta}}\left(\gamma^{M} \lambda\right)_{\alpha}\left(\gamma^{N} \bar{\lambda}\right)_{\widehat{\beta}}}{(\eta \lambda \bar{\lambda})} J^{M} \bar{J}^{N}-\eta_{\alpha \widehat{\beta}} J^{\widehat{\beta}} \bar{J}^{\alpha}\right. \\
\left.-w_{\alpha} \bar{\nabla} \lambda^{\alpha}+\bar{w}_{\widehat{\alpha}} \nabla \bar{\lambda}^{\widehat{\alpha}}-\frac{1}{4} \eta_{[M N][P Q]}\left(w \gamma^{[M N]} \lambda\right)\left(\bar{w} \gamma^{[P Q]} \bar{\lambda}\right)\right] .
\end{gathered}
$$


Note that the second line of (3.4) is identical to the second line of (3.1) whose BRST transformation under

$$
Q+\bar{Q}=\int d z \eta_{\alpha \widehat{\beta}} \lambda^{\alpha} J^{\widehat{\beta}}+\int d \bar{z} \eta_{\alpha \widehat{\beta}} \widehat{\widehat{\lambda}}^{\widehat{\beta}} \bar{J}^{\alpha}
$$

is

$$
\eta_{\alpha \widehat{\beta}}\left(-J^{\widehat{\beta}} \bar{\nabla} \lambda^{\alpha}+\bar{J}^{\alpha} \nabla \bar{\lambda}^{\widehat{\beta}}\right)
$$

Using the transformations

$$
\begin{gathered}
(Q+\bar{Q}) J^{\alpha}=\nabla \lambda^{\alpha}-\eta^{\alpha \widehat{\beta}}\left(\gamma_{M} \bar{\lambda}\right)_{\widehat{\beta}} J^{M} \\
(Q+\bar{Q}) J^{M}=J^{\alpha}\left(\gamma^{M} \lambda\right)_{\alpha}+J^{\widehat{\alpha}}\left(\gamma^{M} \bar{\lambda}\right)_{\widehat{\alpha}} \\
(Q+\bar{Q}) J^{\widehat{\beta}}=\nabla \bar{\lambda}^{\widehat{\beta}}+\eta^{\alpha \widehat{\beta}}\left(\gamma_{M} \lambda\right)_{\alpha} J^{M}
\end{gathered}
$$

and the identity $\gamma_{\widehat{\alpha} \widehat{\beta}}^{M}=\eta_{\alpha \widehat{\alpha}} \eta_{\beta \widehat{\beta}}\left(\gamma^{M}\right)^{\alpha \beta}$, it is easy to verify that the BRST transformation of the first line of (3.4) cancels (3.6), so that $S_{\text {trivial }}$ is BRST-closed. Furthermore, the coefficient $-\frac{1}{2} R^{2}$ multiplying $S_{\text {trivial }}$ has been chosen so that the bonus $U(1)$ transformation of $S_{\text {trivial }}$ cancels (3.3).

Finally, one can show that $S_{\text {trivial }}$ is BRST-trivial by writing it as $S_{\text {trivial }}=Q \bar{Q} \Omega$ where

$\Omega=-\frac{1}{2} R^{2} \int d^{2} z \frac{1}{(\eta \lambda \bar{\lambda})}\left[\frac{1}{4}(w \lambda)(\bar{w} \bar{\lambda})-\frac{1}{8}\left(w \gamma^{M N} \lambda\right)\left(\bar{w} \gamma_{M N} \bar{\lambda}\right)+\frac{1}{4} \frac{\eta^{\alpha \widehat{\beta}}\left(\gamma^{M} \lambda\right)_{\alpha}\left(\gamma^{N} \bar{\lambda}\right)_{\widehat{\beta}}}{(\eta \lambda \bar{\lambda})} J^{M} \bar{J}^{N}\right]$.

To show that $S_{\text {trivial }}=Q \bar{Q} \Omega$, one uses the identity

$$
\delta_{\beta}^{\gamma} \delta_{\alpha}^{\delta}=\frac{1}{2} \gamma_{\alpha \beta}^{M} \gamma_{M}^{\gamma \delta}-\frac{1}{8}\left(\gamma^{M N}\right)_{\alpha}^{\gamma}\left(\gamma_{M N}\right)_{\beta}^{\delta}-\frac{1}{4} \delta_{\alpha}^{\gamma} \delta_{\beta}^{\delta}
$$

together with the BRST transformations of (3.7) and

$$
\begin{gathered}
Q w_{\alpha}=\eta_{\alpha \widehat{\alpha}} J^{\widehat{\alpha}}, \quad \bar{Q} w_{\alpha}=w_{\alpha}^{*}, \\
Q \bar{w}_{\widehat{\alpha}}=\bar{w}_{\widehat{\alpha}}^{*}, \quad \bar{Q} \bar{w}_{\widehat{\alpha}}=\eta_{\alpha \widehat{\alpha}} J^{\alpha}, \\
Q w_{\alpha}^{*}=\eta_{\alpha \widehat{\alpha}}\left(\nabla \bar{\lambda}^{\widehat{\alpha}}-\frac{1}{4} \eta_{[M N][P Q]}\left(w \gamma^{[M N]} \lambda\right)\left(\gamma^{[P Q]} \bar{\lambda}\right)^{\widehat{\alpha}}\right), \quad \bar{Q} w_{\alpha}^{*}=0, \\
Q \bar{w}_{\widehat{\alpha}}^{*}=0, \quad \bar{Q} \bar{w}_{\widehat{\alpha}}^{*}=\eta_{\alpha \widehat{\alpha}}\left(\overline{\nabla \lambda}^{\alpha}+\frac{1}{4} \eta_{[M N][P Q]}\left(\gamma^{[M N]} \lambda\right)^{\alpha}\left(\bar{w} \gamma^{[P Q]} \bar{\lambda}\right)\right) .
\end{gathered}
$$


In reference [6], the auxiliary variables $w_{\alpha}^{*}$ and $\bar{w}_{\alpha}^{*}$ were not included, and the BRST transformations were nilpotent only up to the equations of motion $\nabla \bar{\lambda}^{\widehat{\alpha}}-\frac{1}{4} \eta_{[M N][P Q]}\left(w \gamma^{[M N]} \lambda\right)\left(\gamma^{[P Q]} \bar{\lambda}\right)^{\widehat{\alpha}}=0, \quad \bar{\nabla}^{\alpha}+\frac{1}{4} \eta_{[M N][P Q]}\left(\gamma^{[M N]} \lambda\right)^{\alpha}\left(\bar{w} \gamma^{[P Q]} \bar{\lambda}\right)=0$. Note that $Q w_{\alpha}^{*}$ and $\bar{Q} \bar{w}_{\widehat{\alpha}}^{*}$ are proportional to these equations of motion which come from varying $w_{\alpha}$ and $\bar{w}_{\hat{\alpha}}$. So after adding the term

$$
R^{2} \int d^{2} z \eta^{\alpha \widehat{\beta}} \bar{w}_{\widehat{\beta}}^{*} w_{\alpha}^{*}
$$

to the pure spinor sigma model action of (3.1), the action will be invariant with respect to the BRST transformations of (3.10). The auxiliary variables $w_{\alpha}^{*}$ and $\bar{w}_{\alpha}^{*}$ can be naturally interpreted as antifields which allow the BRST transformation generated by $(Q+\bar{Q})$ to be nilpotent off-shell.

In this construction of a $U(2,2 \mid 4)$-invariant pure spinor sigma model, the only subtlety is the presence of inverse powers of $\left(\eta_{\alpha \widehat{\beta}^{\alpha}} \lambda^{\alpha} \bar{\lambda}^{\widehat{\beta}}\right)$ in $S_{\text {trivial }}$ and $\Omega$. If one Wick-rotates both the $d=2$ and $d=10$ metric to Euclidean space, it is natural to define $\bar{\lambda}_{\alpha} \equiv \eta_{\alpha \widehat{\beta}} \bar{\lambda}^{\widehat{\beta}}$ to be the complex conjugate of $\lambda^{\alpha}$. Using this definition of complex conjugation, $\lambda^{\alpha} \bar{\lambda}_{\alpha}$ is only zero if each component of $\lambda^{\alpha}$ is zero. Therefore, $S_{\text {trivial }}$ and $\Omega$ are well-defined except where $\lambda^{\alpha}=\bar{\lambda}_{\alpha}=0$. As in [8], we shall assume that we can remove the singular point $\lambda^{\alpha}=0$ from the pure spinor space so that $S_{\text {trivial }}$ and $\Omega$ are well-defined.

One possible problem with removing the point $\lambda^{\alpha}=0$ is that, in a flat background, allowing operators such as

$$
\xi=\frac{\theta^{\alpha} \bar{\lambda}_{\alpha}}{(\eta \lambda \bar{\lambda})}
$$

in the Hilbert space implies that the BRST cohomology is trivial. Since $Q \xi=1$, any operator $V$ satisfying $Q V=0$ can be written as $V=Q(\xi V)$. However, $\xi$ of (3.12) is not spacetime supersymmetric, and it was conjectured in [9] that if one restricts operators with poles in $\lambda^{\alpha}$ to spacetime supersymmetric operators (such as the composite $b$ ghost), these operators do not trivialize the Hilbert space.

In the case of an $A d S_{5} \times S^{5}$ background, one can make a similar conjecture with spacetime supersymmetric operators being replaced by $P S U(2,2 \mid 4)$-invariant operators. Since $S_{\text {trivial }}$ and $\Omega$ are $P S U(2,2 \mid 4)$-invariant, the conjecture would imply these operators do not cause problems. Nevertheless, this subtlety certainly deserves further investigation.

3 The structure of the antifields $w_{\alpha}^{*}$ and $\bar{w}_{\alpha}^{*}$ in the pure spinor $A d S_{5} \times S^{5}$ sigma model was also discussed in independent work by Guillaume Boussard [7]]. 


\subsection{Mapping to the A-model}

After adding $S_{\text {trivial }}$ to the pure spinor sigma model action of (3.1), one obtains the $U(2,2 \mid 4)$-invariant action

$$
\begin{gathered}
S=\frac{1}{2} R^{2} \int d^{2} z\left[\frac{1}{2} \frac{\eta^{\alpha \widehat{\beta}}\left(\gamma^{M} \lambda\right)_{\alpha}\left(\gamma^{N} \bar{\lambda}\right)_{\widehat{\beta}}}{(\eta \lambda \bar{\lambda})} \bar{J}^{M} J^{N}-\frac{1}{2} \eta_{\alpha \widehat{\beta}}\left(J^{\widehat{\beta}} \bar{J}^{\alpha}+\bar{J}^{\widehat{\beta}} J^{\alpha}\right)\right. \\
\left.-w_{\alpha} \bar{\nabla} \lambda^{\alpha}+\bar{w}_{\alpha} \nabla \bar{\lambda}^{\widehat{\alpha}}-\frac{1}{4} \eta_{[M N][P Q]}\left(w \gamma^{[M N]} \lambda\right)\left(\bar{w} \gamma^{[P Q]} \bar{\lambda}\right)\right] .
\end{gathered}
$$

It will now be shown that this action is equivalent to the A-model action of (2.7) where $t=\frac{1}{2} R^{2}$.

The first step in relating the actions of (3.13) and (2.7) is to express the supercoset $g \in \frac{P S U(2,2 \mid 4)}{S O(4,1) \times S O(5)}$ in terms of the fermionic coset $G \in \frac{U(2,2 \mid 4)}{U(2,2) \times U(4)}$ and the bosonic variables $Z_{J}^{A}$ and $\bar{Z}_{A}^{J}$. Using the definitions of (2.3) that

$$
Z_{J}^{A}=H_{A^{\prime}}^{A}(x)\left(\tilde{H}^{-1}(\tilde{x})\right)_{J}^{J^{\prime}} \lambda_{J^{\prime}}^{A^{\prime}}, \bar{Z}_{A}^{J}=\left(H^{-1}(x)\right)_{A}^{A^{\prime}} \tilde{H}_{J^{\prime}}^{J}(\tilde{x}) \bar{\lambda}_{A^{\prime}}^{J^{\prime}}
$$

where $H_{A^{\prime}}^{A}(x) \in \frac{S U(2,2)}{S O(4,1)}$ and $H_{J^{\prime}}^{J}(\tilde{x}) \in \frac{S U(4)}{S O(5)}$, it is natural to parameterize $g$ as

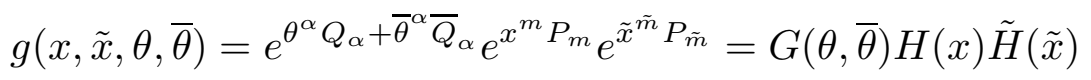

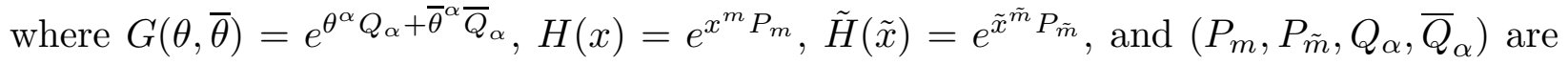
the 10 translation and 32 supersymmetry generators on $A d S_{5} \times S^{5}$.

The map of (3.15) implies that the left-invariant currents $J=g^{-1} \partial g$ which appear in the pure spinor sigma model action are related to $G$ and $H$ as

$$
\begin{gathered}
J_{B^{\prime}}^{A^{\prime}}=\left(H^{-1} \partial H\right)_{B^{\prime}}^{A^{\prime}}+\left(H^{-1}\right)_{A}^{A^{\prime}}\left(G^{-1} \partial G\right)_{B}^{A} H_{B^{\prime}}^{B}, \\
J_{K^{\prime}}^{J^{\prime}}=\left(\tilde{H}^{-1} \partial \tilde{H}\right)_{K^{\prime}}^{J^{\prime}}+\left(\tilde{H}^{-1}\right)_{J}^{J^{\prime}}\left(G^{-1} \partial G\right)_{K}^{J} H_{K^{\prime}}^{K}, \\
J_{J^{\prime}}^{A^{\prime}}=\left(H^{-1}\right)_{A}^{A^{\prime}}\left(G^{-1} \partial G\right)_{J}^{A} \tilde{H}_{J^{\prime}}^{J}, \\
J_{A^{\prime}}^{J^{\prime}}=\left(\tilde{H}^{-1}\right)_{J}^{J^{\prime}}\left(G^{-1} \partial G\right)_{A}^{J} \tilde{H}_{A^{\prime}}^{A} .
\end{gathered}
$$

In terms of the left-invariant currents of (3.1),

$$
J_{B^{\prime}}^{A^{\prime}}=\frac{1}{2} J^{m}\left(\sigma_{m} \sigma_{6}\right)_{B^{\prime}}^{A^{\prime}}+\frac{1}{2} J^{[m n]}\left(\sigma_{m} \sigma_{n}\right)_{B^{\prime}}^{A^{\prime}}
$$




$$
\begin{gathered}
J_{K^{\prime}}^{J^{\prime}}=\frac{1}{2} J^{\tilde{m}}\left(\tilde{\sigma}_{\tilde{m}} \tilde{\sigma}_{6}\right)_{K^{\prime}}^{J^{\prime}}+\frac{1}{2} J^{[\tilde{m} \tilde{n}]}\left(\tilde{\sigma}_{\tilde{m}} \tilde{\sigma}_{\tilde{n}}\right)_{K^{\prime}}^{J^{\prime}}, \\
J_{J^{\prime}}^{A^{\prime}}=\frac{1}{\sqrt{2}}\left[J^{\alpha}\left(f_{\alpha}\right)_{J^{\prime}}^{A^{\prime}}+i J^{\widehat{\alpha}}\left(f_{\widehat{\alpha}}\right)_{B^{\prime}}^{K^{\prime}}\left(\sigma^{6}\right)^{A^{\prime} B^{\prime}} \tilde{\sigma}_{J^{\prime} K^{\prime}}^{6}\right], \\
J_{A^{\prime}}^{J^{\prime}}=\frac{1}{\sqrt{2}}\left[J^{\widehat{\alpha}}\left(f_{\widehat{\alpha}}\right)_{A^{\prime}}^{J^{\prime}}+i J^{\alpha}\left(f_{\alpha}\right)_{K^{\prime}}^{B^{\prime}} \sigma_{A^{\prime} B^{\prime}}^{6}\left(\tilde{\sigma}^{6}\right)^{J^{\prime} K^{\prime}}\right],
\end{gathered}
$$

where $\left(f_{\alpha}\right)_{J^{\prime}}^{A^{\prime}}$ and $\left(f_{\widehat{\alpha}}\right)_{A^{\prime}}^{J^{\prime}}$ are Clebsch-Gordon coefficients for decomposing an $S O(9,1)$ spinor into an $S O(4,1) \times S O(5)$ spinor. Note that $J_{J^{\prime}}^{A^{\prime}}$ has bonus $U(1)$ charge +1 and $J_{A^{\prime}}^{J^{\prime}}$ has bonus $U(1)$ charge -1 , which explains the relative coefficients in (3.17).

The next step in relating the two actions is to use the definitions of $Z_{J}^{A}$ and $\bar{Z}_{A}^{J}$ in (3.14), together with the definitions

$$
Y_{A}^{J}=\left(H^{-1}(x)\right)_{A}^{A^{\prime}} \tilde{H}_{J^{\prime}}^{J}(\tilde{x}) w_{A^{\prime}}^{J^{\prime}}, \quad \bar{Y}_{J}^{A}=H_{A^{\prime}}^{A}(x)\left(\tilde{H}^{-1}(\tilde{x})\right)_{J}^{J^{\prime}} \bar{w}_{J^{\prime}}^{A^{\prime}}
$$

to relate the second lines of (3.13) and (2.7). Since

$$
\begin{gathered}
Y_{A}^{J} \bar{\partial} Z_{J}^{A}=w_{A^{\prime}}^{J^{\prime}} \bar{\partial} \lambda_{J^{\prime}}^{A^{\prime}}+\left(H^{-1} \bar{\partial} H\right)_{B^{\prime}}^{A^{\prime}} w_{A^{\prime}}^{J^{\prime}} \lambda_{J^{\prime}}^{B^{\prime}}-\left(\tilde{H}^{-1} \bar{\partial} \tilde{H}\right)_{J^{\prime}}^{K^{\prime}} w_{A^{\prime}}^{J^{\prime}} \lambda_{K^{\prime}}^{A^{\prime}} \\
=w_{A^{\prime}}^{J^{\prime}} \bar{\partial} \lambda_{J^{\prime}}^{A^{\prime}}+\frac{1}{2}\left(\bar{J}^{m}\left(\sigma_{m} \sigma_{6}\right)_{B^{\prime}}^{A^{\prime}}+\bar{J}^{[m n]}\left(\sigma_{m} \sigma_{n}\right)_{B^{\prime}}^{A^{\prime}}\right)(\lambda w)_{A^{\prime}}^{B^{\prime}} \\
-\frac{1}{2}\left(\bar{J}^{\tilde{m}}\left(\tilde{\sigma}_{\tilde{m}} \tilde{\sigma}_{6}\right)_{K^{\prime}}^{J^{\prime}}+\bar{J}^{[\tilde{m} \tilde{n}]}\left(\tilde{\sigma}_{\tilde{m}} \tilde{\sigma}_{\tilde{n}}\right)_{K^{\prime}}^{J^{\prime}}\right)(w \lambda)_{J^{\prime}}^{K^{\prime}}-\left(G^{-1} \bar{\partial} G\right)_{B}^{A}(Y Z)_{A}^{B}+\left(G^{-1} \bar{\partial} G\right)_{K}^{J}(Y Z)_{J}^{K},
\end{gathered}
$$

one finds that

$$
Y_{A}^{J}(\bar{\nabla} Z)_{J}^{A}=w_{\alpha}\left(\bar{\partial} \lambda+\frac{1}{2} \bar{J}^{[M N]} \gamma_{[M N]} \lambda\right)^{\alpha}+\frac{1}{2} \bar{J}^{m}\left(\sigma_{m} \sigma_{6}\right)_{B^{\prime}}^{A^{\prime}}(\lambda w)_{A^{\prime}}^{B^{\prime}}-\frac{1}{2} \bar{J}^{\tilde{m}}\left(\tilde{\sigma}_{\tilde{m}} \tilde{\sigma}_{6}\right)_{K^{\prime}}^{J^{\prime}}(w \lambda)_{J^{\prime}}^{K^{\prime}} .
$$

Similarly, one finds that

$$
\bar{Y}_{J}^{A}(\nabla \bar{Z})_{A}^{J}=\bar{w}_{\alpha}\left(\partial \bar{\lambda}+\frac{1}{2} J^{[M N]} \gamma_{[M N]} \bar{\lambda}\right)^{\alpha}-\frac{1}{2} J^{m}\left(\sigma_{m} \sigma_{6}\right)_{B^{\prime}}^{A^{\prime}}(\bar{w} \bar{\lambda})_{A^{\prime}}^{B^{\prime}}+\frac{1}{2} J^{\tilde{m}}\left(\tilde{\sigma}_{\tilde{m}} \tilde{\sigma}_{6}\right)_{K^{\prime}}^{J^{\prime}}(\bar{\lambda} \bar{w})_{J^{\prime}}^{K^{\prime}},
$$

and that

$$
(Y Z)_{K}^{J}(\overline{Z Y})_{J}^{K}-(Z Y)_{B}^{A}(\overline{Y Z})_{A}^{B}=(w \lambda)_{K^{\prime}}^{J^{\prime}}(\bar{\lambda} \bar{w})_{J^{\prime}}^{K^{\prime}}-(\lambda w)_{B^{\prime}}^{A^{\prime}}(\bar{w} \bar{\lambda})_{A^{\prime}}^{B^{\prime}}
$$

Putting (3.20)- (3.22) together, one finds that the A-model action of (2.7) is equal to

$$
S=t \int d^{2} z\left[-\frac{1}{2} \eta_{\alpha \widehat{\beta}}\left(J^{\widehat{\beta}} \bar{J}^{\alpha}+\bar{J}^{\widehat{\beta}} J^{\alpha}\right)\right.
$$




$$
\begin{aligned}
& -w_{\alpha}\left(\bar{\partial} \lambda+\frac{1}{2} J^{[M N]} \gamma_{[M N]} \lambda\right)^{\alpha}+\bar{w}_{\alpha}\left(\partial \bar{\lambda}+\frac{1}{2} \bar{J}^{[M N]} \gamma_{[M N]} \bar{\lambda}\right)^{\alpha}-\frac{1}{4} \eta_{[M N][P Q]}\left(w^{[M N]} \lambda\right)\left(\bar{w} \gamma^{[P Q]} \bar{\lambda}\right) \\
& \left.+\frac{1}{2} \eta^{\alpha \beta} w_{\alpha}\left(\gamma_{M} \lambda\right)_{\beta} \bar{J}^{M}+\frac{1}{2} \eta^{\widehat{\alpha} \widehat{\beta}_{\bar{\alpha}}}\left(\gamma_{M} \bar{\lambda}\right)_{\widehat{\beta}} J^{M}-\frac{1}{4}\left(\eta^{\widehat{\alpha} \widehat{\beta}_{\bar{\alpha}}}\left(\gamma_{M} \bar{\lambda}\right)_{\widehat{\beta}}\right)\left(\eta^{\gamma \delta} w_{\gamma}\left(\gamma^{M} \lambda\right)_{\delta}\right)\right] .
\end{aligned}
$$

Under the transformation $\delta w_{\alpha}=\left(\lambda \gamma^{M}\right)_{\alpha} \Lambda_{M}$, the first two lines of (3.23) are invariant, but the last line transforms as

$$
\delta S=t \int d^{2} z \Lambda^{N} \eta^{\alpha \beta}\left(\gamma_{N} \lambda\right)_{\alpha}\left(\gamma_{M} \lambda\right)_{\beta}\left(\frac{1}{2} \bar{J}^{M}-\frac{1}{4} \eta^{\widehat{\gamma \delta}} \bar{w} \widehat{\gamma}\left(\gamma^{M} \bar{\lambda}\right)_{\widehat{\delta}}\right)
$$

Since $\delta S=0$ onshell, one learns that the equations of motion for $w_{\alpha}$ imply that (3.24) vanishes for any $\Lambda^{N}$, which implies that

$$
\left(\gamma_{M} \lambda\right)_{\beta}\left(\bar{J}^{M}-\frac{1}{2} \eta^{\widehat{\gamma} \widehat{\delta}_{\bar{w}}} \widehat{\gamma}_{\gamma}\left(\gamma^{M} \bar{\lambda}\right)_{\widehat{\delta}}\right)=0
$$

Similarly, the equations of motion for $\bar{w}_{\alpha}$ imply that

$$
\left(\gamma_{M} \bar{\lambda}\right)_{\beta}\left(J^{M}-\frac{1}{2} \eta^{\gamma \delta} w_{\gamma}\left(\gamma^{M} \lambda\right)_{\delta}\right)=0
$$

Since the equations of motion of (3.25) and (3.26) are auxiliary, they can be plugged back into the action of (3.23). One finds that all three terms in the last line of (3.23) are proportional to each other, and their sum is equal to

$$
t \int d^{2} z \frac{1}{2} \frac{\eta^{\alpha \widehat{\beta}}\left(\gamma_{M} \lambda\right)_{\alpha}\left(\gamma_{N} \bar{\lambda}\right)_{\widehat{\beta}}}{(\eta \lambda \bar{\lambda})} \bar{J}^{M} J^{N}
$$

which coincides with the first term in (3.13). So the A-model action of (2.7) is equal to

$$
\begin{aligned}
S= & t \int d^{2} z\left[\frac{1}{2} \frac{\eta^{\alpha \widehat{\beta}}\left(\gamma^{M} \lambda\right)_{\alpha}\left(\gamma^{N} \bar{\lambda}\right)_{\widehat{\beta}}}{(\eta \lambda \bar{\lambda})} \bar{J}^{M} J^{N}-\frac{1}{2} \eta_{\alpha \widehat{\beta}}\left(J^{\widehat{\beta}} \bar{J}^{\alpha}+\bar{J}^{\widehat{\beta}} J^{\alpha}\right)\right. \\
& \left.-w_{\alpha} \bar{\nabla} \lambda^{\alpha}+\bar{w}_{\alpha} \nabla \bar{\lambda}^{\alpha}-\frac{1}{4} \eta_{[M N][P Q]}\left(w \gamma^{[M N]} \lambda\right)\left(\bar{w} \gamma^{[P Q]} \bar{\lambda}\right)\right]
\end{aligned}
$$

which coincides with the $U(2,2 \mid 4)$-invariant pure spinor sigma model of (3.13) when $t=$ $\frac{1}{2} R^{2}$. 


\subsection{BRST operator}

For the A-model action of (2.4) and (2.7), the obvious guesses for left and right-moving BRST operators are the scalar generators of $\mathrm{N}=2$ worldsheet supersymmetry,

$$
\int d z Z_{J}^{A}\left(G^{-1} \partial G\right)_{A}^{J}, \quad \int d \bar{z} \bar{Z}_{A}^{J}\left(G^{-1} \bar{\partial} G\right)_{J}^{A}
$$

Surprisingly, these do not match the left and right-moving BRST operators in the pure spinor sigma model and would therefore give the incorrect cohomology.

Under the map of (3.15), it is easy to check that (3.29) map into the operators

$$
\int d z \lambda^{\alpha}\left(\eta_{\alpha \widehat{\beta}} J^{\widehat{\beta}}+i \eta_{\alpha \beta} J^{\beta}\right), \quad \int d \bar{z} \bar{\lambda}^{\widehat{\beta}}\left(\eta_{\widehat{\alpha} \widehat{\beta}} \bar{J}^{\widehat{\alpha}}-i \eta_{\alpha \widehat{\beta}} J^{\alpha}\right) .
$$

However, the pure spinor left and right-moving BRST operators are

$$
Q=\int d z \eta_{\alpha \widehat{\beta}^{\alpha}} \lambda^{\alpha} J^{\widehat{\beta}}, \quad \bar{Q}=\int d \bar{z} \eta_{\alpha \widehat{\beta}} \bar{\lambda}^{\widehat{\beta}} J^{\alpha}
$$

So to reproduce the correct cohomology, one must map the left and right-moving BRST operators of (3.31) into the A-model variables, which implies

$$
\begin{gathered}
Q=\int d z Z_{J}^{A}\left[\left(G^{-1} \partial G\right)_{A}^{J}+i\left(H^{-1}\right)_{A}^{A^{\prime}} \tilde{H}_{J^{\prime}}^{J} \sigma_{A^{\prime} B^{\prime}}^{6}\left(\tilde{\sigma}^{6}\right)^{J^{\prime} K^{\prime}}\left(H^{-1}\right)_{B}^{B^{\prime}} \tilde{H}_{K^{\prime}}^{K}\left(G^{-1} \partial G\right)_{K}^{B}\right], \\
\bar{Q}=\int d \bar{z}_{A}^{J}\left[\left(G^{-1} \partial G\right)_{J}^{A}+i H_{A^{\prime}}^{A}\left(\tilde{H}^{-1}\right)_{J}^{J^{\prime}}\left(\sigma^{6}\right)^{A^{\prime} B^{\prime}} \tilde{\sigma}_{J^{\prime} K^{\prime}}^{6} H_{B^{\prime}}^{B}\left(\tilde{H}^{-1}\right)_{K}^{K^{\prime}}\left(G^{-1} \partial G\right)_{B}^{K}\right],
\end{gathered}
$$

where $H$ and $\tilde{H}$ are defined in terms of $Z$ and $\bar{Z}$ by the inverse map of (2.3).

Note that after adding the BRST-trivial term of (3.4) to the pure spinor action, both

$$
\eta_{\alpha \widehat{\beta}} \lambda^{\alpha} J^{\widehat{\beta}} \text { and } \eta_{\alpha \beta} \lambda^{\alpha} J^{\beta}
$$

are holomorphic currents. This is easy to see since the action of (3.13) is $U(1)$ invariant, and $J^{\widehat{\alpha}}$ and $J^{\alpha}$ transform into each other under this $U(1)$. Furthermore, one can check that the currents in (3.33) are nilpotent and satisfy the OPE

$$
\left(\eta_{\alpha \widehat{\beta}} \lambda^{\alpha} J^{\widehat{\beta}}(y)\right) \quad\left(\eta_{\gamma \delta} \lambda^{\gamma} J^{\delta}(z)\right) \rightarrow(y-z)^{-2} \eta_{\alpha \beta} \lambda^{\alpha}(y) \lambda^{\beta}(z)
$$

So the operators of (3.33) satisfy the OPE's of the two spin-one fermionic generators, $G^{+}$ and $\tilde{G}^{+}$, of a twisted "small" $\mathrm{N}=4$ superconformal algebra whose generators are

$$
\left[T, G^{+}, \tilde{G}^{+}, G^{-}, \tilde{G}^{-}, J^{++}, J, J^{--}\right]
$$


It is easy to explicitly construct the generators

$$
\begin{gathered}
G^{+}=\eta_{\alpha \widehat{\beta}} \lambda^{\alpha} J^{\widehat{\beta}}, \quad \tilde{G}^{+}=\eta_{\alpha \beta} \lambda^{\alpha} J^{\beta}, \quad J^{++}=\eta_{\alpha \beta} \lambda^{\alpha} \lambda^{\beta}, \\
J=\lambda^{\alpha} w_{\alpha}, \quad T=\frac{1}{2} \eta_{M N} J^{M} J^{N}+\eta_{\alpha \widehat{\beta}} J^{\widehat{\beta}} J^{\alpha}-w_{\alpha}\left(\partial \lambda+\frac{1}{2} J^{[M N]} \gamma_{M N} \lambda\right)^{\alpha},
\end{gathered}
$$

however, the remaining $\mathrm{N}=4$ generators do not appear to be easy to construct. For example, the obvious guess for $J^{--}$is $J^{--}=\eta^{\alpha \beta} w_{\alpha} w_{\beta}$, but this is not holomorphic. Also, to construct $G^{-}$and $\tilde{G}^{-}$, one would need the analog of the composite $b$ ghost in the pure spinor formalism which is not easy to construct even in a flat background.

Nevertheless, the existence of an "almost" $\mathrm{N}=4$ superconformal algebra for the Amodel with the generators of (3.35) allows the construction of the nilpotent left and rightmoving BRST operators of (3.32) which differ from the naive guess of (3.29). In other words, the existence of an "almost" $\mathrm{N}=4$ algebra allows the choice of

$$
Q=\int d z\left(A G^{+}+B \tilde{G}^{+}\right)
$$

where $A$ and $B$ are arbitrary constants. The naive guess of (3.29) corresponds to $B=i A$, whereas the map of the pure spinor BRST operators of (3.32) corresponds to $B=0$.

Since the bonus $U(1)$ symmetry is preserved only if $A^{2}+B^{2}=0$, it seems reasonable to conjecture that any choice of the constants $A$ and $B$ is allowed as long as $A^{2}+B^{2}$ is nonzero. If this conjecture is correct, the cohomology of (3.36) should be independent of $A$ and $B$, except for the singular choice where $A^{2}+B^{2}=0$.

\section{Worldsheet derivation of Chern-Simons/topological gravity duality}

In this section we will review the worldsheet derivation of the duality between the A-model topological string on the resolved conifold and Chern-Simons $U(N)$ gauge theory on $S^{3}$ [3], along the lines proposed in [2].

The basic idea is to start from the closed string side, i.e. the topological A-model on the resolved conifold, with $t$ being the modulus of $\mathbf{P}^{1}$. One then considers expanding the A-model sigma model near the $t \rightarrow 0$ limit, as a perturbation in $t$. However, the non-linear sigma model is singular in this limit. Instead one considers the gauged linear sigma model formulation of the conifold where the Higgs branch of the $U(1)$ gauge system flows in the IR to the non-linear sigma model of the resolved conifold. The gauged linear sigma model is not singular in this regime, and the fact that the geometry is singular translates in this 
formulation to the opening up of a new branch for the gauge theory: the Coulomb branch [10]. In other words the operation of going to the IR and going to the non-linear sigma model formulation do not commute in this limit and we end up with a completion of the worldsheet theory using the gauged linear sigma model.

In the limit as $t \rightarrow 0$ the path-integral of the worldsheet theory will have two regions. One region is in the Higgs branch and the other in the Coulomb branch. Moreover the degrees of freedom which are light in the Higgs branch are massive in the Coulomb branch. In the UV the separation between these regions is not sharp. But as we take the IR limit the separation becomes sharper. Indeed we have to take the IR limit as the CFT description of the worldsheet is what arises in string perturbation theory.

We thus end up with a worldsheet marked by $H$ and $C$ regions. However, it turns out that if the topology of the $C$ region is anything but a disc, then the amplitude vanishes [3]. This is because the contribution of each $C$ region to the path integral ends up being a total derivative in the moduli. In particular if $\theta$ denotes the angular part of $t$, the contribution of each $C$ region is given as

$$
\oint d \theta \frac{\partial F}{\partial \theta}
$$

This is manifestly zero except when $F$ is not a single valued function of $\theta$. This is only the case for the disc topology where the partition function of a topological string is not well defined (due to the $S L(2, R)$ symmetry) 1 . We thus end up with the Higgs branch and a number of Coulomb branches, all of which are in disc topology. Moreover, since the fields in the Higgs branch are massive in the Coulomb branch, in the IR they vanish as they approach the Coulomb branch. In other words the discs play the role of holes with D-brane boundary condition for the Higgs branch. Moreover one can show that up to a BRST trivial deformation (deforming the $t=0$ conifold to the deformed conifold) these can be viewed as Lagrangian D-branes living on $S^{3}$. Moreover doing the path-integral on each Coulomb branch yields a factor of $t$ which is identified with $t=N \lambda$. In particular this is the right structure for it to correspond to $N$ Lagrangian D-brane insertions on $S^{3}$. This makes contact with Witten's formulation of Chern-Simons theory as an A-model with $N$ D-branes wrapping Lagrangian submanifolds [11]. We thus end up with a description of the theory in terms of a $U(N)$ Chern-Simons gauge theory.

\footnotetext{
4 There are potentially a few other possibilities, but these can be ruled out [3].
} 


\subsection{Operator/State Correspondence}

In the context of the large $N$ limit of Chern-Simons theory, no local gravitation operators exist. However in other applications, such as in the AdS/CFT context we are studying here, there are local deformations on both sides and one needs to map them. It is well known that the dictionary of AdS/CFT relates a given state on the gravity side to an operator on the gauge theory side. Here we would like to comment that the fact that this is a one to one map fits naturally in the context of the [3] derivation of large $N$ duality. Namely consider deforming the gravitational side by a vertex operator corresponding to a scattering state. In the limit of $t \rightarrow 0$ we ask where would the vertex operator lie. It should be that they all lie in the Coulomb branch. In other words it should be that in the Higgs branch at $t=0$, cohomology is trivial. Moreover it should be that for each Coulomb branch region at most one vertex operator should be present deforming the boundary conditions on the D-branes, which can be viewed as operator insertions from the gauge theory perspective. If there were non-vanishing contributions coming from 2 or more vertex operators on the Coulomb branch then the 1-1 correspondence between states and boundary operators will not work. The approach of [3] is indeed compatible with this idea, because the A-model contribution on the disc with no insertion or 1 insertion is ambiguous (because there are residual conformal symmetries). So in the computation of $\oint d F$ they could lead to contributions. However, for 2 or more insertions of vertex operators in the C-region, $F$ is well defined and thus $\oint d F=0$.

With this review we are now ready to study the case of interest in this paper and see how much of this structure carries over.

\section{Gauged Linear Sigma Model and Zero Radius Limit}

In proving the open-closed duality which relates $d=3$ Chern-Simons theory and the resolved conifold, it was useful to write the topological A-model for the resolved conifold as a gauged linear sigma model where the parameter $t$ multiplying the A-model action is the Fayet-Illiopoulos modulus in the gauged linear sigma model. In the limit where $t \rightarrow 0$, the gauged linear sigma model for the resolved conifold can develop both a Coulomb phase and a Higgs phase, and the Coulomb phase was interpreted as D-brane holes which describe the faces in Feynman diagrams of the Chern-Simons gauge theory. 
In this section, we suggest that a similar technique may be useful for obtaining a worldsheet derivation of the Maldacena conjecture which relates $\mathcal{N}=4 \mathrm{~d}=4$ super-YangMils and the $A d S_{5} \times S^{5}$ sigma model. In section (5.1), we shall write the A-model action of (2.4) as a gauged linear sigma model with a $U(4)$ worldsheet gauge field. And in section (5.2), we shall argue that in the limit where $t \rightarrow 0$, a Coulomb phase develops which can be interpreted as D-brane holes. Furthermore, it will be argued that these D-brane holes are associated with gauge-invariant $\mathcal{N}=4 \mathrm{~d}=4$ super-Yang-Mills operators. Finally, a possible connection with the twistorial formulation of $\mathcal{N}=4 \mathrm{~d}=4$ super-Yang-Mills will be discussed in subsection (5.3).

\subsection{Gauged linear sigma model}

The A-model action of (2.4) is based on the coset $\frac{U(2,2 \mid 4)}{U(2,2) \times U(4)}$ which can be interpreted as a "fermionic" version of the Grassmannian $\frac{U(M+N)}{U(M) \times U(N)}$. As shown in [12] [13], the nonlinear sigma model action based on this Grassmannian can be written as a gauged linear sigma model by introducing either a $U(M)$ or a $U(N)$ worldsheet gauge field, together with an appropriate set of matter fields transforming in the fundamental representation of the gauge group. For the coset $\frac{U(2,2 \mid 4)}{U(2,2) \times U(4)}$, we shall choose to introduce a $U(4)$ worldsheet gauge field, however, we suspect that the alternative choice of introducing a $U(2,2)$ gauge field would not affect our conclusions.

In two-dimensional $\mathrm{N}=(2,2)$ superspace, the $U(4)$ worldsheet gauge field is described by the real prepotential,

$$
V_{S}^{R}\left(z, \bar{z}, \kappa^{+}, \kappa^{-}, \bar{\kappa}^{+}, \bar{\kappa}^{-}\right)
$$

where $R, S=1$ to 4 are local $U(4)$ indices, and the matter fields are described by the chiral and antichiral superfields,

$$
\Phi_{R}^{\Sigma}\left(z, \bar{z}, \kappa^{+}, \kappa^{-}\right), \quad \bar{\Phi}_{\Sigma}^{R}\left(z, \bar{z}, \bar{\kappa}^{+}, \bar{\kappa}^{-}\right),
$$

where $\Sigma=(A, J)$ is a global $U(2,2 \mid 4)$ index and, as in the previous sections, $A=1$ to 4 is a global $U(2,2)$ index and $J=1$ to 4 is a global $U(4)$ index. Note that the matter fields transform in the fundamental representation of the gauge group and that $\Phi_{R}^{A}$ is a fermionic superfield whereas $\Phi_{R}^{J}$ is a bosonic superfield.

The gauged linear sigma model action is easily written in $U(2,2 \mid 4)$-invariant notation as

$$
S=\int d^{2} z \int d^{4} \kappa\left[\bar{\Phi}_{\Sigma}^{S}\left(e^{V}\right)_{S}^{R} \Phi_{R}^{\Sigma}-t V_{R}^{R}\right]
$$


where $t$ is a constant parameter multiplying the Fayet-Illiopoulos term. When $t$ is nonzero, this action is easily shown to be equivalent to the A-model action of (2.4) by solving the equations of motion for the preprotential $V_{R}^{S}$. The equation of motion for $V_{S}^{R}$ is

$$
t \delta_{S}^{R}=\left(e^{V}\right)_{T}^{R} \bar{\Phi}_{\Sigma}^{T} \Phi_{S}^{\Sigma}
$$

which implies that

$$
V_{S}^{R}=\delta_{S}^{R} \log t-\log \left(\bar{\Phi}_{\Sigma}^{R} \Phi_{S}^{\Sigma}\right)
$$

Plugging this auxiliary equation of motion into (5.1), one finds

$$
S=t \int d^{2} z \int d^{4} \kappa T r\left[\log \left(\bar{\Phi}_{\Sigma}^{R} \Phi_{S}^{\Sigma}\right)\right]
$$

Assuming that $\Phi_{R}^{J}$ and $\bar{\Phi}_{J}^{R}$ are invertible matrices, one can define the chiral and antichiral superfields $\Theta_{J}^{A}$ and $\bar{\Theta}_{A}^{J}$ as

$$
\Theta_{J}^{A} \equiv \Phi_{R}^{A}\left(\Phi_{R}^{J}\right)^{-1}, \quad \bar{\Theta}_{A}^{J} \equiv \bar{\Phi}_{A}^{R}\left(\bar{\Phi}_{J}^{R}\right)^{-1}
$$

and write the action as

$$
S=t \int d^{2} z \int d^{4} \kappa T r\left[\log \left(\delta_{K}^{J}+\bar{\Theta}_{A}^{J} \Theta_{K}^{A}\right)+\log \left(\Phi_{R}^{J}\right)+\log \left(\bar{\Phi}_{J}^{R}\right)\right]
$$

Since $\log \left(\Phi_{R}^{J}\right)$ is chiral and $\log \left(\bar{\Phi}_{J}^{R}\right)$ is antichiral, the second and third terms of (5.5) vanish and the action coincides with (2.4).

\subsection{Zero radius limit}

As shown in [14], the gauged linear sigma model is very convenient for studying the limit where $t \rightarrow 0$. Since $t$ is identified with $\frac{1}{2} R^{2}$ where $R$ is the $A d S_{5} \times S^{5}$ radius, this limit corresponds to small 't Hooft coupling where perturbative $\mathcal{N}=4 \mathrm{~d}=4$ super-Yang-Mills is a good description of the theory. In this $t \rightarrow 0$ limit, it will be shown that the closed string variables in the gauged linear sigma model can exist either in the Higgs phase where the $U(4)$ gauge symmetry is broken, or in the Coulomb phase where the $U(4)$ gauge symmetry is unbroken.

To analyze the different phases, we shall focus on the worldsheet fields with zero conformal weight since only these fields can obtain nonzero expectation values. After 
performing an A-twist, the only field with zero conformal weight in the prepotential $V_{R}^{S}$ is the complex field $\sigma_{R}^{S}$ where, in Wess-Zumino gauge,

$$
V_{R}^{S}=\sigma_{R}^{S} \kappa_{+} \bar{\kappa}_{-}+\bar{\sigma}_{R}^{S} \kappa_{-} \bar{\kappa}_{+}+\left(A_{z}\right)_{R}^{S} \kappa_{+} \bar{\kappa}_{+}+\left(A_{\bar{z}}\right)_{R}^{S} \kappa_{-} \bar{\kappa}_{-}+\ldots
$$

And after an A-twist, the only matter fields with zero conformal weight are $\left(\phi_{R}^{\Sigma}, \psi_{R}^{\Sigma}\right)$ and $\left(\bar{\phi}_{\Sigma}^{R}, \bar{\psi}_{\Sigma}^{R}\right)$ where

$$
\Phi_{R}^{\Sigma}=\phi_{R}^{\Sigma}+\kappa_{+} \psi_{R}^{\Sigma}+\ldots, \quad \bar{\Phi}_{\Sigma}^{R}=\bar{\phi}_{\Sigma}^{R}+\bar{\kappa}_{-} \bar{\psi}_{\Sigma}^{R}+\ldots
$$

Note that $\left(\phi_{R}^{J}, \psi_{R}^{A}, \bar{\phi}_{J}^{R}, \bar{\psi}_{A}^{R}\right)$ are bosonic fields and $\left(\phi_{R}^{A}, \psi_{R}^{J}, \bar{\phi}_{A}^{R}, \bar{\psi}_{J}^{R}\right)$ are fermionic fields.

If one sets to zero all component fields with nonzero conformal weight, the equation of motion of (5.2) for $V_{R}^{S}$ implies that the remaining fields satisfy

$$
\phi_{R}^{\Sigma} \bar{\phi}_{\Sigma}^{S}=\delta_{R}^{S} t, \quad \phi_{R}^{\Sigma} \bar{\psi}_{\Sigma}^{S}=0, \quad \psi_{R}^{\Sigma} \bar{\phi}_{\Sigma}^{S}=0, \quad \psi_{R}^{\Sigma} \bar{\psi}_{\Sigma}^{S}=t \sigma_{R}^{S}
$$

When $t$ is nonzero, the first equation of (5.8) implies that one can gauge $\phi_{R}^{J}=\delta_{R}^{J} \sqrt{t}$ up to terms which are quadratic in the fermionic fields $\phi_{R}^{A}$. The second and third equations of (5.8) define the fermionic fields $\psi_{R}^{J}$ and $\bar{\psi}_{J}^{R}$ in terms of $\left(\phi_{R}^{A}, \psi_{R}^{A}\right)$ and $\left(\bar{\phi}_{A}^{R}, \bar{\psi}_{A}^{R}\right)$. And the fourth equation implies that $\sigma_{R}^{S}$ is fixed to satisfy $\sigma_{R}^{S}=t^{-1} \psi_{R}^{\Sigma} \bar{\psi}_{\Sigma}^{S}$. Since $U(4)$ symmetry is broken and $\sigma_{R}^{S}$ is fixed, this is the Higgs phase. In this phase, the unconstrained variables $\left(\phi_{R}^{A}, \psi_{R}^{A}\right)$ and $\left(\bar{\phi}_{A}^{R}, \bar{\psi}_{A}^{R}\right)$ are related to the nonlinear sigma model variables $\left(\theta_{J}^{A}, Z_{J}^{A}\right)$ and $\left(\bar{\theta}_{A}^{J}, \bar{Z}_{A}^{J}\right)$ using the identification of (5.4).

When $t \rightarrow 0$, one possibility is that the worldsheet variables stay in the Higgs phase. In this phase, $\left(\phi_{R}^{A}, \psi_{R}^{A}\right)$ and $\left(\bar{\phi}_{A}^{R}, \bar{\psi}_{A}^{R}\right)$ are unconstrained, and $\sigma_{R}^{S}$ is constrained to satisfy $\sigma_{R}^{S}=t^{-1} \psi_{R}^{\Sigma} \bar{\psi}_{\Sigma}^{S}$ (which generically will diverge). However, another possibility when $t \rightarrow 0$ is that $\sigma_{R}^{S}$ is unconstrained, but the matter variables are constrained to satisfy

$$
\phi_{R}^{\Sigma} \bar{\phi}_{\Sigma}^{S}=0, \quad \phi_{R}^{\Sigma} \bar{\psi}_{\Sigma}^{S}=0, \quad \psi_{R}^{\Sigma} \bar{\phi}_{\Sigma}^{S}=0, \quad \psi_{R}^{\Sigma} \bar{\psi}_{\Sigma}^{S}=0
$$

This phase will be called the Coulomb phase since the Cartan subgroup of $U(4)$ symmetry is unbroken and $\sigma_{R}^{S}$ is unconstrained. The fields $\phi_{R}^{\Sigma}, \psi_{R}^{\Sigma}$ are massive in this branch and can be integrated out. If one Wick-rotates the $U(2,2 \mid 4)$ signature to a $U(4 \mid 4)$ signature, then the equations of (5.9) imply that

$$
\phi_{R}^{\Sigma}=\psi_{R}^{\Sigma}=\bar{\phi}_{\Sigma}^{R}=\bar{\psi}_{\Sigma}^{R}=0
$$

This solution is analogous to the Coulomb phase for the resolved conifold where all the matter fields are forced to vanish. So if one works in the Euclidean signature of $U(4 \mid 4)$, the 
regions in the Coulomb phase appear as "holes" in the closed string worldsheet. As in [3], it is natural to identify the boundary of these holes with open string D-branes corresponding to the "faces" of the gauge theory Feynman diagrams.

However, unlike in the conifold/Chern-Simons duality, one expects in the AdS/YangMills duality that regions in the Coulomb phase carry additional information corresponding to the different gauge-invariant super-Yang-Mills operators. In other words, the D-brane holes can be described as boundary states in the closed string theory, and the physical closed string vertex operators for these boundary states should correspond to gaugeinvariant super-Yang-Mills operators. As will now be shown, if one constructs solutions to the Coulomb phase equations of (5.9) using the original Minkowski space signature of $U(2,2 \mid 4)$, one can easily describe the half BPS super-Yang-Mills operators. This corresponds to the special case where the boundary conditions on the D-brane have no spatial derivative. It is still an open question how to describe the non-BPS super-Yang-Mills operators.

To construct solutions to (5.9) in Minkowski space signature, it will be convenient to split the $S U(2,2)$ index $A$ as $A=(a, \dot{a})$ where $a, \dot{a}=1$ to 2 , and to split the $S U(4)$ index $J$ as $J=\left(j, j^{\prime}\right)$ where $j, j^{\prime}=1$ to 2 . Furthermore, define $\bar{\Phi}_{A}^{J}$ to be the "harmonic" conjugate of $\Phi_{J}^{A}$ where "harmonic conjugation" switches the $a$ and $\dot{a}$ representations and also switches the $j$ and $j^{\prime}$ representations. Note that harmonic conjugation is equivalent to complex conjugation multiplied by a $Z_{2}$ transformation in $S U(4)$, and is commonly used for defining superfields in harmonic superspace.

With this definition of harmonic conjugation, it is easy to see that

$$
\phi_{R}^{j}=\psi_{R}^{j}=\phi_{R}^{a}=\psi_{R}^{a}=0, \quad \bar{\phi}_{j^{\prime}}^{R}=\bar{\psi}_{j^{\prime}}^{R}=\bar{\phi}_{\dot{a}}^{R}=\bar{\psi}_{\dot{a}}^{R}=0
$$

is a solution of (5.9) which breaks $U(2,2 \mid 4)$ invariance to a $U(1,1 \mid 2) \times U(1,1 \mid 2)$ subgroup. By deforming the solution of (5.11) using the $\frac{U(2,2 \mid 4)}{U(1,1 \mid 2) \times U(1,1 \mid 2)}$ parameters

$$
\left[x_{\dot{a}}^{a}, \theta_{j^{\prime}}^{a}, \bar{\theta}_{\dot{a}}^{j}, u_{j^{\prime}}^{j}\right]
$$

one discovers that the most general solution of (5.9) is

$$
\begin{aligned}
& \phi_{R}^{j}=u_{j^{\prime}}^{j} \phi_{R}^{j^{\prime}}+\bar{\theta}_{\dot{a}}^{j} \phi_{R}^{\dot{a}}, \quad \psi_{R}^{j}=u_{j^{\prime}}^{j} \psi_{R}^{j^{\prime}}+\bar{\theta}_{\dot{a}}^{j} \psi_{R}^{\dot{a}}, \\
& \phi_{R}^{a}=\theta_{j^{\prime}}^{a} \phi_{R}^{j^{\prime}}+x_{\dot{a}}^{a} \phi_{R}^{\dot{a}}, \quad \psi_{R}^{a}=\theta_{j^{\prime}}^{a} \psi_{R}^{j^{\prime}}+x_{\dot{a}}^{a} \psi_{R}^{\dot{a}},
\end{aligned}
$$




$$
\begin{array}{cc}
\bar{\phi}_{j^{\prime}}^{R}=u_{j^{\prime}}^{j} \bar{\phi}_{j}^{R}+\theta_{j^{\prime}}^{a} \bar{\phi}_{a}^{R}, & \bar{\psi}_{j^{\prime}}^{R}=u_{j^{\prime}}^{j} \bar{\psi}_{j}^{R}+\theta_{j^{\prime}}^{a} \bar{\psi}_{a}^{R}, \\
\bar{\phi}_{\dot{a}}^{R}=\bar{\theta}_{\dot{a}}^{j} \bar{\phi}_{j}^{R}+x_{\dot{a}}^{a} \bar{\phi}_{a}^{R}, & \bar{\psi}_{\dot{a}}^{R}=\bar{\theta}_{\dot{a}}^{j} \bar{\psi}_{j}^{R}+x_{\dot{a}}^{a} \bar{\psi}_{a}^{R},
\end{array}
$$

where $\left(x_{\dot{a}}^{a}, u_{j^{\prime}}^{j}\right)$ and $\left(\theta_{j^{\prime}}^{a}, \bar{\theta}_{\dot{a}}^{j}\right)$ are eight bosonic and eight fermionic parameters, and $\left(\phi_{R}^{j^{\prime}}, \psi_{R}^{j^{\prime}}, \phi_{R}^{\dot{a}}, \psi_{R}^{\dot{a}}\right)$ and $\left(\bar{\phi}_{j^{\prime}}^{R}, \psi_{j^{\prime}}^{R}, \phi_{\dot{a}}^{R}, \psi_{\dot{a}}^{R}\right)$ are unconstrained.

The parameters of (5.12) are precisely the projective harmonic superspace variables used in [15] and [16] to describe $\mathcal{N}=4 \mathrm{~d}=4$ super-Yang-Mills operators. As shown in [15], the $U(2,2 \mid 4)$ generators can be expressed in terms of these parameters as

$$
\begin{aligned}
& M_{U}^{U^{\prime}}=\frac{\partial}{\partial y_{U^{\prime}}^{U}}, \quad M_{U}^{V}=y_{U^{\prime}}^{V} \frac{\partial}{\partial y_{U^{\prime}}^{U}}-C \delta_{U}^{V}, \quad M_{U^{\prime}}^{V^{\prime}}=-y_{U^{\prime}}^{V} \frac{\partial}{\partial y_{V^{\prime}}^{V}}+C \delta_{U^{\prime}}^{V^{\prime}}, \\
& M_{U^{\prime}}^{U}=-y_{U^{\prime}}^{V} y_{V^{\prime}}^{U} \frac{\partial}{\partial y_{V^{\prime}}^{V}}+2 y_{U^{\prime}}^{U} C
\end{aligned}
$$

where $U=(a, j), U^{\prime}=\left(\dot{a}, j^{\prime}\right), y_{U^{\prime}}^{U}=\left(x_{\dot{a}}^{a}, \theta_{j^{\prime}}^{a}, \bar{\theta}_{\dot{a}}^{j}, u_{j^{\prime}}^{j}\right)$, and $C$ is a central charge which commutes with the $U(2,2 \mid 4)$ generators. Note that the $U(1,1 \mid 2) \times U(1,1 \mid 2)$ generators $M_{U}^{V}$ and $M_{U^{\prime}}^{V^{\prime}}$ act linearly on the harmonic variables of (5.12).

In the Coulomb phase, one cannot define (5.4) and there is no connection between the linear sigma model variables and the pure spinor variables of (3.13). So the only sensible definition of the BRST operator in the Coulomb phase are the usual $\mathrm{N}=2$ worldsheet supersymmetry generators of the gauged linear sigma model which transform the fields of zero conformal weight as

$$
Q \phi_{R}^{\Sigma}=\psi_{R}^{\Sigma}, \quad \overline{Q \phi}_{\Sigma}^{R}=\bar{\psi}_{\Sigma}^{R}
$$

Although this BRST transformation preserves the full $U(2,2 \mid 4)$ invariance, there is no contradiction when $t=\frac{1}{2} R^{2} \rightarrow 0$ since, in this limit, the super-Yang-Mills theory has no interaction terms which means it contains the bonus $U(1)$ symmetry.

When the worldsheet variables are in the Coulomb phase, it is easy to verify from the solution of (5.13) that $Q \phi_{R}^{j^{\prime}}=\psi_{R}^{j^{\prime}}, Q \phi_{R}^{\dot{a}}=\psi_{R}^{\dot{a}}, \overline{Q \phi}_{j}^{R}=\bar{\psi}_{j}^{R}, \overline{Q \phi}_{a}^{R}=\bar{\psi}_{a}^{R}$, and the harmonic variables of (5.12) are BRST invariant. Therefore, any function of $\left[x_{\dot{a}}^{a}, \theta_{j^{\prime}}^{a}, \bar{\theta}_{\dot{a}}^{j}, u_{j^{\prime}}^{j}\right]$ which is independent of $\left(\phi_{R}^{j^{\prime}}, \psi_{R}^{j^{\prime}}, \phi_{R}^{\dot{a}}, \psi_{R}^{\dot{a}}, \bar{\phi}_{j^{\prime}}^{R}, \psi_{j^{\prime}}^{R}, \phi_{\dot{a}}^{R}, \psi_{\dot{a}}^{R}\right)$ is in the BRST cohomology.

But as was shown in [16], supergravity solutions on $A d S_{5} \times S^{5}$ with $C-2$ units of $S^{5}$ angular momentum are in one-to-one corespondence with functions of $\left[x_{\dot{a}}^{a}, \theta_{j^{\prime}}^{a}, \bar{\theta}_{\dot{a}}^{j}, u_{j^{\prime}}^{j}\right]$ with a given central charge $C$. So these functions in the BRST cohomology describe the half BPS gauge-invariant super-Yang-Mills operators. For example, if $V_{C}$ is a function with central charge $C$, then $V_{C}=1$ corresponds to the super-Yang-Mills state $\operatorname{Tr}\left(Z^{C}\right)$ where 
$Z$ is the scalar with $U(1)$ charge +1 with respect to $M_{j}^{j}$ of (5.14). The other half BPS states with $C$ super-Yang-Mills fields can be obtained from $V_{C}=1$ by hitting with the generators $M_{U^{\prime}}^{U}$ of (5.14).

So at $t=0$ in the Coulomb phase, functions in the BRST cohomology describe the half BPS gauge-invariant super-Yang-Mills operators at zero coupling. Up to now, only constant modes of the worldsheet variables in the Coulomb phase have been considered. It is plausible that non-BPS gauge-invariant super-Yang-Mills operators will be described by functions which also depend on non-constant modes. This seems natural since half BPS operators are related to "massless" closed string vertex operators which only depend on constant modes, whereas non-BPS operators are related to "massive" closed string vertex operators which depend on non-constant modes of the worldsheet variables.

\subsection{Twistorial formulation}

It is quite intriguing that the gauged linear sigma model we are studying is closely related to the twistor space relevant for the formulation of $\mathcal{N}=4$ Yang-Mills [17]. In particular consider the limit $t \rightarrow 0$ and focus on the Coulomb branch of our $U(4)$ gauged linear sigma model in this limit. A generic point on the Coulomb branch corresponds to breaking the gauge group to $U(1)^{4} / S_{4}$ where $S_{4}$ is the permutation group on the four $U(1)$ factors. In this case the charged matter multiplet for each $U(1)$ corresponds to a (4|4) space. Thus the gauged linear sigma model will give rise to the Coulomb branch of the topological A-model of four copies of $\mathbf{C P}^{3 \mid 4}$, one for each $U(1)$. In other words the corresponding non-linear sigma model in its Higgs branch is

$$
\operatorname{Sym}^{\otimes 4}\left(\mathbf{C P}^{3 \mid 4}\right)
$$

Note that the above construction is related to the well-known geometric fact that the Grassmannian $\frac{U(n+m)}{U(n) \times U(m)}$ can be viewed as $S y m^{\otimes m}\left(\mathbf{C P}^{m+n-1}\right)$ [13.

The appearance of the twistor space in our context is rather interesting and suggests perhaps another view of our link to gauge theory. In fact the twistor space does seem to play a role in the boundary conditions we have found since, as discussed in [15], twistors and harmonic variables are related when the fields are onshell. As explained in [15], the onshell equations for a function $f(y)$ where $y_{U^{\prime}}^{U}=\left[x_{\dot{a}}^{a}, \theta_{j^{\prime}}^{a}, \bar{\theta}_{\dot{a}}^{j}, u_{j^{\prime}}^{j}\right]$ are

$$
\frac{\partial}{\partial y_{\left[U^{\prime}\right.}^{[U}} \frac{\partial}{\partial y_{\left.V^{\prime}\right]}^{V]}} f(y)=0
$$


which implies the mass-shell condition $\frac{\partial}{\partial x^{a a}} \frac{\partial}{\partial x_{a \dot{a}}} f(y)=0$. The onshell equations of (5.16) can be easily solved by writing the Penrose-like transform

$$
f(y)=\int d \zeta \tilde{f}\left(\zeta_{U}, \zeta_{U^{\prime}} \equiv \zeta_{U} y_{U^{\prime}}^{U}\right)
$$

where $\left(\zeta_{U}, \zeta_{U^{\prime}}\right)$ are $\mathbf{C P}^{3 \mid 4}$ twistor variables and $\tilde{f}(\zeta)$ is a twistor function of the appropriate $U(1)$ weight.

However there is a difference between the appearance of twistors here and the one in [17]: In that case one was dealing with the topological B-model, whereas here we are dealing with the topological A-model. In fact the situation here is more similar to the setup considered in 18] where the open A-model topological string on $\mathbf{C P}^{3 \mid 4}$ was proposed to be a perturbative realization of $\mathcal{N}=4 \mathrm{YM}$. This could be related by S-duality to the formulation of [17]. It could also be that the A-model and B-model theory can appear similar in a hyperkahler setup as is the case in [19]. It would be interesting to explore the connection with twistor space further.

\section{Conclusions and Open Problems}

We have taken a step towards a worldsheet derivation of the Maldacena conjecture. In particular we have argued that the A-model topological string on $\frac{U(2,2 \mid 4)}{U(2,2) \times U(4)}$, which describes string theory in the background of $A d S_{5} \times S^{5}$, is a gauged linear sigma model that in the small radius limit develops a new branch, the Coulomb branch, which creates 'holes' on the worldsheet. This should correspond to the open string diagrams describing $\mathcal{N}=4$ supersymmetric Yang-Mills. As evidence for this we showed that the half BPS operators of the gauge theory naturally arise as solutions to the boundary conditions of the Coulomb branch.

There are a number of things that need to be better understood. One has to analyze the effect of integrating out the degrees of freedom on the Coulomb branch and show that they give rise to the factor $N \lambda$ as is expected from the Chan-Paton factors. More generally one would like to show, in addition to the half BPS states that we discussed, how the precise dictionary between gravitational states and gauge theory operators work.

We have found an intriguing connection to (four copies of) the twistor space. This is very suggestive and calls for a deeper understanding of the role of twistors in the worldsheet derivation of the Maldacena conjecture. 


\section{Acknowledgment}

We would like to thank O. Aharony, R. Gopakumar, P. Howe, J. Maldacena, L. Motl, H. Ooguri, M. Roček, W. Siegel, B.C. Vallilo and E. Witten for valuable discussions. We would also like to thank the 5th Simons Workshop in Mathematics and Physics and the Strings 2007 Conference for their hospitality. The research of N.B. was supported in part by CNPq grant 300256/94-9 and FAPESP grant 04/11426-0. The research of C.V. was supported in part by NSF grants PHY-0244821 and DMS-0244464. 


\section{References}

[1] J. Maldacena, The Large N Limit of Superconformal Field Theories and Supergravity, Adv. Theor. Math. Phys. 2 (1998) 231, hep-th/9711200.

[2] R. Gopakumar and C. Vafa, On the Gauge Theory/Geometry Correspondence, Adv. Theor. Math. Phys. 3 (1999) 1415, hep-th/9811131.

[3] H. Ooguri and C. Vafa, Worldsheet Derivation of a Large N Duality, Nucl. Phys. B641 (2002) 3, hep-th/0205297.

[4] N. Berkovits, H. Ooguri and C. Vafa, On the Worldsheet Derivation of Large N Dualities for the Superstring, Comm. Math. Phys. 252 (2004) 259, hep-th/0310118.

[5] N. Berkovits, New Limit of the $A d S_{5} \times S^{5}$ Sigma Model, JHEP 0708 (2007) 011, hep-th/0703282.

[6] N. Berkovits, Quantum Consistency of the Superstring in $A d S_{5} \times S^{5}$ Background, JHEP 0503 (2005) 041, hep-th/0411170.

[7] G. Boussard, private communication.

[8] N. Nekrasov, Lectures on Curved Beta-Gamma Systems, hep-th/0511008.

[9] N. Berkovits, Relating the RNS and Pure Spinor Formalisms for the Superstring, JHEP 0108 (2001) 026, hep-th/0104247.

[10] E. Silverstein and E. Witten, Criteria for Conformal Invariance of (0,2) Models, Nucl. Phys. B444 (1995) 161, hep-th/9503212.

[11] E. Witten, Chern-Simons Gauge Theory as a String Theory, Prog. Math. 133 (1995) 637, hep-th/9207094.

[12] U. Lindstrom and M. Roček, Scalar-Tensor Duality and N=1, N=2 Non-Linear Sigma Models, Nucl. Phys. B222 (1983) 285.

[13] S. Cecotti and C. Vafa, On Classification of N=2 Supersymmetric Theories, Comm. Math. Phys. 158 (1993) 569, hep-th/9211097.

[14] E. Witten, Phases of N=2 Theories in Two Dimensions, Nucl. Phys. B403 (1993) 159, hep-th/9301042.

[15] M. Hatsuda and W. Siegel, A New Holographic Limit of $A d S_{5} \times S^{5}$, Phys. Rev. D67 (2003) 066005, hep-th/0211184.

[16] P. Heslop and P.S. Howe, Chiral Superfields in IIB Supergravity, Phys. Lett. B502 (2001) 259, hep-th/0008047.

[17] E. Witten, Perturbative Gauge Theory as a String Theory in Twistor Space, Comm. Math. Phys. 252 (2004) 189, hep-th/0312171.

[18] A. Neitzke and C. Vafa, N=2 Strings and the Twistorial Calabi-Yau, hep-th/0402128.

[19] A. Kapustin and E. Witten, Electric-Magnetic Duality and the Geometric Langlands Program, hep-th/0604151. 